\title{
La protección social como problema de coordinación
}

\author{
Eleonora Nun* \\ Aldo Madariaga**
}

\begin{abstract}
Resumen
El objetivo de este artículo es aportar nuevos elementos al debate sobre cómo analizar el desempeño de las políticas de protección social, con especial atención al caso chileno. Se desarrolla el concepto coordinación y sus alcances en el ámbito de la protección social, generando un marco analítico que permite poner atención en tres aspectos críticos: la generación de expectativas y motivaciones entre los beneficiarios; la articulación de las agencias relacionadas con la producción de las prestaciones de protección social y la vinculación de los recursos sistémicos necesarios para producir dichas prestaciones. Estos elementos son operacionalizados en una matriz mediante la cual se ordena el análisis y observación del desempeño de las políticas y programas en distintos aspectos de su funcionamiento.
\end{abstract}

Palabras clave: Política social - protección social - desempeño - coordinación.

\begin{abstract}
This article aims to provide new insights to the debate about how to analyze the performance of social protection policies, with special interest in Chile. The article develops the concept of coordination and its reach in the field of social protection, generating an analytical framework that draws attention to three critical aspects: the generation of expectations and motivations between beneficiaries; the articulation of the agencies related with the production of social protection benefits, and the linkage of the systemic resources necessary to produce those benefits. These elements are translated into a matrix which helps ordinating the analysis and observing the performance of policies and programmes in different aspects of its functioning.
\end{abstract}

Keywords: Social policy - social protection - performance - coordination.

* Socióloga, Universidad de Chile. Se ha desempeñado como asistente de investigación en el Equipo de Desarrollo Humano del PNUD y en la División de Desarrollo Social de la CEPAL. E-mail: e leonoranun@ gmail.com.

** Sociólogo, Universidad de Chile. Diplomado en Análisis de Políticas, Facultad de Economía de la misma Universidad. Se ha desempeñado como asistente de investigación en la División de Desarrollo Social de la CEPAL. E-mail: aldomadariaga@gmail.com. 


\section{LOS DESAFÍOS DE LA POLÍTICA SOCIAL EN EL NUEVO MILENIO}

Chile ha sido históricamente pionero en América Latina en el ámbito de la política social (Filgueira y Filgueira, 2002). Desde la década de 1920 con la incorporación de la ley de seguro social y hasta 1970, las reformas se orientaron a incrementar la cobertura y la variedad de las prestaciones de un sistema de protección social en constante ampliación (Riesco, 2009; Schkolnick y Bonnefoy, 1994) ${ }^{1}$. A partir de 1980, sin embargo, este es reformulado introduciéndose nuevos criterios como es la focalización del gasto social en los sectores más empobrecidos y la incorporación de actores privados en la provisión de bienes públicos (Ibíd). Con el retorno de la democracia en 1990, el Estado vuelve a asumir un rol significativo en el ámbito de la protección social, esta vez de mitigación de las fallas del mercado y de regulación y supervigilancia de las acciones privadas (Ibíd).

Como efecto de la última estrategia, a partir de 1990 se observa una disminución continuada de los índices de pobreza e indigencia en el país (Larrañaga y Herrera, 2008). Una importante inversión fiscal y diversas reformas institucionales en un contexto de crecimiento económico sostenido contribuyeron a estos resultados (Ffrench-Davis, 2003; Stallings, 2001). No obstante, ya desde mediados de la década estos indicadores comienzan a enfrentar mayores dificultades para seguir mejorando, especialmente aquellos que dicen relación con la erradicación de la pobreza extrema y la disminución de la desigualdad (Ibíd). Esto hace evidente el hecho que la fórmula que permitió la inclusión social de parte importante de la población en un primer momento no es suficiente o adecuada al pasar a una etapa difícil de la modernización. Se habla así de un "efecto dual del crecimiento" (Torche, 1996) que se manifiesta en un mejoramiento de las condiciones de vida de amplios sectores de la población que coincide con la creciente disminución de posibilidades de quienes tienen menos capacidades de integración.

Esta tendencia está en el origen de las reformas que se emprendieron a partir del año 2000 con el objetivo de reintroducir elementos de tipo solidario y estatal en diversos componentes del sistema de protección, como el sistema de salud, el de previsión social y la reformulación de las políticas y programas asistenciales. Durante este último periodo, además, se observa un importante cambio en la orientación de la política social. Comienza a instalarse la idea que la política social debe dejar de ser un conjunto de prestaciones para pobres y pasar a ser un instrumento de construcción de ciudadanía.

El presente artículo nace de la siguiente hipótesis de trabajo: pese a las innovaciones emprendidas durante los últimos años en materia de política social, persiste una mirada que no siempre le permite aprehender la naturaleza compleja de los desafíos que enfrenta. Esta complejidad radicaría en el hecho que en la actualidad su núcleo problemático es la

1 Si bien el presente artículo utiliza algunas referencias sobre teoría de sistemas y aplica ciertas categorías asociadas a esta tradición, la noción de "sistema de protección social" es utilizada en su acepción más corriente y extendida en la literatura sobre el tema, referida al conjunto de acciones, procesos y estructuras involucrados en la función de inclusión social (Cf. Herrera y Castón, 2003). 
coordinación de relaciones sociales en torno a la definición de un estatus de ciudadanía y ya no el ejercicio de un rol compensatorio ante carencias específicas.

Para indagar en esta problemática el artículo propone un ejercicio analítico consistente en proponer una definición de coordinación aplicable al análisis de las políticas sociales y su desempeño. La definición dada centra su atención en tres aspectos o niveles analíticos del problema ${ }^{2}$. Primero, en el cómo ofrecer a los beneficiarios expectativas en torno a su participación en el sistema de protección que sean motivantes a nivel individual, y que al mismo tiempo se correspondan con las definiciones socialmente construidas en relación al problema de la inclusión -nivel microsocial. Segundo, en la articulación de los distintos agentes, acciones y prestaciones que dan forma al sistema de protección -nivel funcional. Tercero, en la vinculación de las expectativas y las operaciones funcionales con los recursos sistémicos que marcan los limites a su realización -nivel sistémico.

En la medida que el foco se pone en la política social como construcción de ciudadanos sujetos de derechos (Abramovich, 2006), la coordinación efectuada a través de la protección social centra su problemática en el vínculo entre los beneficiarios y el sistema de protección. De este modo, a diferencia de las evaluaciones de impacto o las consideraciones en torno a la eficiencia en la asignación del gasto, que se centran en variables sistémicas -uso eficiente de los recursos- o funcionales -ejecución en los plazos de los productos previstos-, la evaluación del desempeño del sistema de protección y de sus componentes desde la perspectiva aquí propuesta pone el acento en el cumplimiento de las expectativas de inclusión que el sistema promueve entre sus beneficiarios.

El artículo se organiza en tres partes. Primero se recogen los antecedentes que motivaron la investigación, poniendo especial énfasis en las transformaciones que ha experimentado la sociedad chilena durante las últimas décadas y que han tenido como consecuencia una complejización del escenario en el que se enmarca el problema de la protección social. En un segundo momento se construye, a partir de los aportes teóricos desarrollados por diversas corrientes, una definición de coordinación adecuada y útil para la comprensión de la política social. Esta parte concluye con la elaboración de una matriz de indicadores que facilita el análisis de casos específicos de políticas sociales desde la problemática de la coordinación. Finalmente se reflexiona brevemente en torno a los aportes de esta mirada

2 La diferenciación en tres niveles analíticos tiene una amplia gama de orígenes y de usos en la historia de la sociología. Por ejemplo, Max Weber trabajó con la distinción entre acción individual, órdenes institucionales, como las iglesias y los Estados, y órdenes de valor (la ciencia, el arte, la política, la economía, entre otros). Buena parte de la sociología de inspiración weberiana trabajó la distinción entre estos niveles analíticos. La teoría de sistemas, por su parte, trabaja con la distinción entre sistemas sociales, organizaciones y sistemas psíquicos. La distinción aquí planteada surge del trabajo de Jessop (1999), quien intenta combinar la teoría de sistemas (no luhmanniana) con las teorías institucionalistas más cercanas a la sociología clásica. De este modo el autor propone tres niveles de incrustación de la organización social (embeddedness of social organization), un nivel social de las relaciones interpersonales, un nivel institucional de las relaciones interorganizacionales y un nivel societal de órdenes institucionales funcionalmente diferenciados. Los niveles han sido adecuados al presente estudio y se abordan con más detalle en el punto 3.2. 
para la comprensión del desempeño de la política social a partir del ejemplo específico del sistema de pensiones.

\section{PROBLEMAS COMPLEJOS QUE DEMANDAN SOLUCIONES COMPLEJAS. LAS RAÍCES PROFUNDAS DE LOS DESAFÍOS}

La explicación del nuevo escenario que enmarca la política social no debe buscarse únicamente en la política social misma. También, y de manera más sustantiva, en las transformaciones experimentadas por la sociedad chilena en las últimas décadas. Se puede hacer referencia a transformaciones en los tres planos mencionados anteriormente: sistémico, funcional y microsocial.

\subsection{La tensión entre autonomía e interdependencia en el nivel sistémico}

Las sociedades complejas cuentan entre sus principales características con la creciente especialización funcional de los distintos órdenes institucionales en que están fundadas. Este fenómeno, en las ciencias sociales, se conoce como "diferenciación funcional" y una de sus principales consecuencias es que los ámbitos funcionalmente diferenciados se autonomizan hasta tal punto que ninguno de ellos es capaz de comunicarse con los demás sin salirse de su propia lógica de funcionamiento.

En momentos anteriores en Chile, el Estado por medio de la política podía decidir hegemónicamente sobre el funcionamiento de ámbitos como la educación, la salud o la economía, sobre quién y cómo se integraba a la sociedad. En la actualidad estas son problemáticas en cuya resolución intervienen una diversidad de lógicas -eficiencia económica, legitimidad política, trayectorias culturales. Dado que ningún sistema tiene la capacidad de imponerse por sobre los demás, sólo pueden aspirar a un ajuste espontáneo de sus operaciones recíprocas. La coordinación, como observa Lechner, "habría de ser internalizada al interior de cada subsistema. Los subsistemas se coordinarían entre sí mediante ajustes internos, que asimilarían las señales y turbulencias externas. La coordinación quedaría incorporada pues a la autorregulación" (Lechner, 1997: 13). Este proceso, sin embargo, entraña también la pretensión de universalidad de los códigos particulares de cada sistema, con lo que se genera una competencia por la colonización de otros ámbitos que no han logrado diferenciarse completamente.

En el caso de la política social, estos intentos de colonización han dado origen a la coexistencia de distintas lógicas o normas de funcionamiento en las prestaciones ofrecidas (técnicas, económicas, políticas) que no necesariamente encuentran una solución de continuidad (Cf. Christensen y Lægreid, 2005). Ello puede observarse, por ejemplo, en las particulares formas de inclusión social que promueven los distintos espacios de política y programas sociales. Están, por ejemplo, aquellos que promueven una inclusión a la "nación", enfatizando sus aspectos culturales y simbólicos como la política educacional. Otros privilegian una forma de inclusión "económica" por medio del incremento directo de los recursos monetarios de las personas como las transferencias de ingresos (pensiones 
y subsidios). Están por otro lado, los que se orientan a propiciar, simultáneamente, una inclusión en diversas dimensiones de la "ciudadanía", como es el Sistema de Protección Social Chile Solidario.

El fenómeno que aquí se describe conlleva una importante paradoja. Los sistemas van especializándose en el cumplimiento de determinadas funciones y, sin embargo, cada vez más las problemáticas sociales atraviesan una multiplicidad de ámbitos, lógicas y dinámicas. Por ejemplo, debates propios del momento actual como son los temas medioambientales involucran no sólo consideraciones respecto de la ecología, sino que están cruzados por intereses y reivindicaciones económicas, políticas, culturales e identitarias, entre otros. De acuerdo con esto, ningún sistema por sí mismo tiene la capacidad para absorber, procesar y solucionar problemas de naturaleza cada vez más compleja, presionando por la generación de mecanismos capaces de coordinar el funcionamiento de los distintos sistemas y sus prestaciones específicas en torno a problemáticas comunes como requiere la protección social.

\subsection{La entrada en juego de nuevos actores en el nivel funcional}

Durante los años noventa se realizaron esfuerzos que apuntaban a generar un marco legal e institucional que permitiera dotar de coherencia al conjunto de actores, instituciones y prestaciones que conforman el sistema de protección social con el fin de cumplir con las expectativas de inclusión contenidas en este.

Crecientemente, sin embargo, se fue haciendo evidente la distancia entre los propósitos y los diseños de los programas, y entre estos y las realidades institucionales o sociales en las que intervenían, lo que no permitía generar los resultados esperados (Raczynski, 2008). Entre los problemas más agudos Raczynski menciona la debilidad en la interfase donde el programa o los servicios se encuentran con la población beneficiaria, y la pertinencia de las acciones, esto es, su adecuación a la diversidad de requerimientos de la política social. En un intento por abordar estos problemas, se observa paralelamente un proceso creciente de diversificación del número y tipo de actores que participan del diseño e implementación de políticas y programas sociales que impacta tanto en los elementos de desempeño propiamente tal como en aquellos de gestión de la misma.

El desafío de coordinación se instaló, así, en la necesidad de regular la coexistencia de distintos agentes y lógicas en la provisión de bienes y servicios sociales. Esto es, generar una modalidad que permita dotar de coherencia al conjunto de instituciones y prestaciones que conforman el sistema de protección social. Buena parte de esto es lo que intenta el sistema de protección social lanzado por el gobierno a inicios del 2000. No obstante ello, persisten una serie de dificultades.

En primer lugar para coordinar las acciones e intereses de los nuevos actores involucrados en la prestación (públicos y privados, con y sin fines de lucro). Como proponen Marcel y Rivera "La incorporación de otros actores a la entrega de prestaciones sociales ha generado una constelación de nuevos problemas asociados a la dificultad para dirigir la acción de 
actores privados, con intereses y visiones propias, hacia el logro de objetivos de política pública" (2008: 154). Junto con esto, al traspasarse parte de la responsabilidad de la gestión de programas sociales a municipios o gobiernos regionales, surge la necesidad de coordinar los niveles centrales y locales de la administración pública.

En segundo lugar, la coexistencia al interior del sistema de protección de una diversidad de programas, cada uno con sus propios objetivos, destinatarios, instrumentos, prestadores, financiamiento, etc., supone una mayor complejidad a la hora de organizar y darle coherencia al conjunto del sistema en términos de la información disponible y la institucionalidad requerida.

Adicionalmente, la idea que predominó en períodos anteriores según la cual los beneficiarios de la política social constituían individuos con necesidades que debían ser asistidas, hoy en día está siendo reemplazada por una lógica de derechos que los concibe como sujetos con el derecho de exigir a la sociedad determinadas prestaciones y servicios (Abramovich, 2006). Esta orientación supone un importante desafío a la hora de coordinar las políticas sectoriales con aquellas focalizadas a los sectores vulnerables de la población.

Finalmente, cada vez más los propios usuarios son incorporados al proceso de diseño, monitoreo y evaluación de los programas sociales de los que son beneficiarios. Se busca que los agentes sean protagonistas de las soluciones entregadas. Esto implica incorporar mayor complejidad a la solución porque ahora no es sólo el proveedor y su racionalidad los que intervienen, sino también la de los beneficiarios, sus intereses y motivaciones.

En la medida que crece la complejidad de la política social y de su gestión, crece también la necesidad de racionalización de su función ante el aumento y complejización de las demandas. Esto se relaciona directamente con el siguiente punto, esto es, el impacto de los procesos de individualización.

\subsection{Una sociedad más demandante en el nivel microsocial}

Durante los últimos años se han producido importantes transformaciones culturales en Chile. Retomando los planteamientos del PNUD (2000, 2002, 2009), una de las más importantes es la profundización de los procesos de individualización. A partir de éstos, las trayectorias biográficas pasan a depender cada vez más de las decisiones, orientaciones y pautas de vida individuales. Los metarrelatos e instituciones que anteriormente cumplieron la función de dotar de un sentido las trayectorias privadas a partir de una normatividad pública, hoy han perdido lugar frente a la fuerza de la reivindicación personal: "Individualización significa que cada persona debe definir por sí misma las elecciones, valores y relaciones que hacen su proyecto de vida. Es el resultado de la valoración social de la autonomía personal, de la pérdida de autoridad de las tradiciones y del aumento de alternativas en los modos de vida" (PNUD, 2002: 189).

Este fenómeno tiene importantes consecuencias referidas al tipo de demanda que la ciudadanía formula hacia el Estado y en términos más generales, hacia la organización 
social como sistema de generación y distribución de recursos. En la actualidad, estas demandas se articulan en torno a las condiciones que hacen posible la realización de los proyectos de vida individuales, de modo que la oferta de productos y servicios deja de venir determinada por el productor y su propia interpretación de las necesidades de los usuarios o consumidores, y pasa a depender de las preferencias y elecciones de estos últimos. A nivel de las políticas sociales, por ejemplo, los subsidios a la demanda y la incorporación de agentes privados que diversifican la oferta de servicios, dan cuenta de este fenómeno.

Como conclusión de todo lo anterior, puede decirse que la política social se enfrenta a escenarios más complejos en distintos planos. Esta complejidad radicaría en los procesos de diferenciación funcional e interdependencia sistémica y la dificultad que representan a la hora de procesar demandas y coordinar respuestas desde todos los ámbitos en los que éstas se inscriben. También en las transformaciones experimentadas en el nivel funcional donde por la diversidad de actores y la variedad de prestaciones ofrecidas, se requiere de mecanismos que permitan articular todo esto en un conjunto coherente. Finalmente, en los cambios ocurridos en la configuración cultural del país donde procesos como la individualización hacen recaer sobre la política social demandas cada vez más exigentes desde sus beneficiarios.

En este contexto, los problemas que enfrenta la política social dejan de referirse a la carencia de recursos financieros, infraestructura o vacíos institucionales. Estos son entendidos como problemas de baja complejidad porque son "fácilmente delimitables, se dispone de abundante información, capacidad de control y consenso respecto de lo que hay que hacer" (PNUD, 2009: 41). Se observa más bien que la política social se enfrenta a problemáticas que dicen relación con la coordinación de relaciones sociales complejas, tanto en el ámbito de la provisión de prestaciones, como a nivel de las expectativas a ser satisfechas entre los beneficiarios. Estos constituyen "ámbitos muy densos en relaciones y significados sociales, donde están presentes actores con relativa autonomía y los centros de control son múltiples y difusos (...). Su núcleo problemático radica en la organización de las relaciones entre actores" (Ibíd: 43). Por lo mismo, estos problemas para ser abordados requieren de estrategias que intervengan a nivel de las interacciones entre agentes, es decir, en espacios dinámicos más que sobre variables estáticas. Como se verá a continuación, ello puede abordarse como un problema de coordinación social.

\section{EL PROBLEMA DE LA COORDINACIÓN COMO ENTRADA ANALÍTICA}

La noción de coordinación social remite al problema general de las formas de constitución de un orden social. Esto es, de cómo por medio de la acción de determinados mecanismos en los distintos espacios sociales se seleccionan los motivos de los agentes individuales y se guían sus operaciones de manera estable y relativamente predecible. Estos mecanismos resuelven el problema de la doble contingencia al reducir el número de posibilidades que enfrentan los agentes en cada situación, a la vez que permiten reforzar el reconocimiento mutuo de las operaciones recíprocas. 
Un mecanismo de coordinación corresponde al conjunto de dispositivos institucionales que operacionaliza determinados principios normativos en pautas de acción concretas. De este modo, al querer llevar a cabo alguna acción en determinado ámbito, los agentes se encuentran con un espacio social poblado de definiciones previas y elementos que permiten reducir la carga informativa necesaria para tomar sus decisiones. De esta manera se van generando pautas habitualizadas de interacción.

En la actualidad, la centralidad del problema de la coordinación social está evidenciada en las transformaciones mencionadas. Éstas han tenido como consecuencia la pérdida de eficacia de los horizontes normativos compartidos para la integración de las perspectivas de los diversos agentes. En este contexto, se entiende la coordinación social como "la posibilidad que dos instancias (personas, sistemas, instituciones, estructuras) que se reconocen como distintas puedan generar un conjunto de conexiones de sentido y de acción, identificable y delimitado. En cuanto tal, ese conjunto requiere que las conexiones sean estabilizadas recíprocamente y que se disponga de medios para asegurar una relativa variación armónica" (Millán, 1999: 63).

\subsection{Mecanismos de coordinación social e instituciones}

\section{i) Los mecanismos de coordinación traducen ciertos principios normativos}

Los mecanismos de coordinación incorporan principios normativos bajo la forma de expectativas estructuradas social y temporalmente $(\mathrm{Ibi}$ id $)$. De acuerdo con esto, un mecanismo de coordinación define las expectativas posibles de ser cumplidas en el espacio y tiempo que el mismo delimita. Estas expectativas, por lo demás, generan motivaciones para que los agentes participen del espacio de interacción propuesto. De no considerarse que la acción concertada producirá los resultados esperados, y sobre todo que producirá resultados a los que no podría llegarse de manera individual, entonces la coordinación no se produce.

Una dimensión particularmente crítica del desempeño de los mecanismos de coordinación es precisamente su capacidad para generar expectativas que más allá de la situación específica de quienes participan, sean normativamente vinculantes. En términos generales, puede decirse que este principio representa el valor que la coordinación social misma tiene para la conformación de un orden social: más allá de los principios específicos que sustenten un determinado mecanismo, la principal expectativa contenida en un ámbito de coordinación es la de la coordinación misma. Esto quiere decir que estos mecanismos deben orientarse no sólo a "elevar el grado de realización de expectativas de cada participante, sino sobre todo a mantener firme la expectativa general de una coordinación probable de los cursos de acción individual, incluso más allá del resultado" (Ibíd).

Esta expectativa general debe mantenerse vigente como principio de orientación incluso en casos en que no logre cumplirse. Para esto, los mecanismos de coordinación recurren al derecho. Éste representa una estructura de expectativas que posee un importante grado de estabilidad más allá de la particularidad de las situaciones. Permite, por lo tanto, neutralizar el efecto desestabilizador de situaciones en que las expectativas no son cumplidas. 
Dado que sobre el derecho pesa una aceptación generalizada, su capacidad para coordinar es menos sensible a las variaciones en el entorno (de expectativas o recursos) que aquellos mecanismos que no operan por esta vía, como es el caso de las convenciones sociales. Por ejemplo, en tanto mecanismo de coordinación, una política social se apoya en el derecho por una parte para estabilizar expectativas y, por otra, para hacer vinculantes sus orientaciones en todos los niveles en los que interviene. "La producción de decisiones vinculantes, generadas mediante procedimientos aceptados, es un mecanismo de coordinación que se instituye bajo la forma de legalidad" (Ibíd: 70). En este caso específico, si la legalidad es el recurso institucional para la coordinación, el fundamento normativo es la capacidad del Estado para representar los intereses colectivos.

No todos los mecanismos de coordinación se apoyan en el derecho para hacer vinculantes sus propuestas, ya sea por su espontaneidad o informalidad. Éstos, sin embargo, también se sustentan en una normatividad que permite integrar las expectativas de coordinación ofrecidas y los resultados observados en discursos estructurados y coherentes. Operan justificando los estados resultantes y manteniendo la legitimidad de su utilización típica en determinados contextos sociales. La legitimidad de las pautas de acción emana, más que del tipo de orientaciones normativas que contienen, del hecho práctico de ser utilizadas por y ser reproducidas en los intercambios sociales y de permitir coordinar efectivamente un conjunto de relaciones con una alta probabilidad. De esta manera, además de convocar a los participantes, el sustento normativo de los mecanismos de coordinación legitima las pautas de acción ofrecidas.

\section{ii) Los mecanismos de coordinación se codifican en matrices institucionales}

Para operar en la práctica, los mecanismos de coordinación son codificados en matrices institucionales que gobiernan un determinado ámbito o espacio social específico. Éstas operan como agregado de normas, leyes y reglas formales e informales que delimitan las posibilidades de acción.

El que en un ámbito predomine uno u otro mecanismo de coordinación depende de cuál sea el objeto de la coordinación así como de las formas históricas de su institucionalización. En este proceso se producen variaciones respecto de la forma típica ideal producto de las propias trayectorias institucionales que limitan las alternativas posibles (path-dependence) y de las complementariedades positivas y negativas que suponen los otros ámbitos institucionales (Amable, 2003). Además, las instituciones así cristalizadas dependen de las estrategias que despliegan distintos grupos en tanto constituyen soluciones de economía política. Esto significa que en la cristalización de una matriz institucional influyen pugnas de poder entre grupos por los beneficios distributivos de la misma, de modo que estas deben entenderse en términos de un equilibrio o compromiso de intereses (Amable 2003; Esping-Andersen, 1989).

\subsection{Los tres niveles de la coordinación}

Como ya se adelantaba, pueden distinguirse tres niveles analíticos interconectados a partir de los cuales observar la constitución de la realidad social desde el punto de vista de 
la coordinación: sistemas, instituciones y agentes. Cuando los problemas de coordinación de estos niveles son considerados en su conjunto, puede referirse a ellos como un problema de governance, esto es, de cómo "guiar múltiples agencias, instituciones y sistemas que son al mismo tiempo, operacionalmente autónomos los unos de los otros, y estructuralmente acoplados a través de varias formas de interdependencia recíproca" (Jessop, 1999: 1, traducción nuestra).

A nivel de las agencias, las instituciones de coordinación social actúan estableciendo expectativas que construyen un vínculo entre la acción actual y aquellas anteriores y posteriores. La coordinación en un espacio social se produce cuando se acepta su pretensión de validez -adecuación a los fines individuales que orientan la acción-y legitimidad-probabilidad de ser seguida por un conjunto de actores. En relación a esto, las expectativas tienden a actualizar maneras pasadas y probadas de resolver la contingencia, actualizándolas en función de las posibilidades que define cada nueva interacción -o espacio de coordinación-, proceso que podría ser definido como un "aprendizaje social".

A nivel funcional, los mecanismos de coordinación actúan sobre la organización de un conjunto de prácticas sociales y de operaciones o prestaciones sistémicas en el marco de un determinado problema social. Las diversas expectativas de coordinación inscritas en un ámbito funcional no siempre son coherentes entre sí. Dado que en un mismo ámbito coexisten instituciones formales (reglamentos, leyes, constituciones) e informales (normas sociales, convenciones), públicas y privadas, que rigen para un gran número de personas o sólo para un conjunto pequeño, la armonización de criterios y normas de funcionamiento requieren de un esfuerzo explícito que, en el marco de las políticas sociales, se entiende típicamente como de planificación. A decir de Garnier (2000: 15), en este caso la coordinación "no se reduce simplemente a 'hacer compatibles' unas acciones gubernamentales con otras, a hacer compatibles unos programas con otros, de manera que se eviten tanto las duplicidades y desperdicios como los conflictos y choques que pueden ocurrir cuando se dan traslapes no planeados entre ellos, o cuando esa misma carencia de coordinación deja espacios vacíos en la acción pública (...) de lo que se trata es de coordinar las políticas de manera que se logre integrar las distintas decisiones y acciones de gobierno dentro de una sola visión de conjunto y con una clara perspectiva de largo plazo".

Finalmente, a nivel sistémico la problemática de la coordinación involucra la discusión respecto de los elementos comunicativos que permiten enlazar un conjunto de sistemas relativamente autónomos e interdependientes a los requerimientos funcionales específicos. Los mecanismos de coordinación en este nivel se orientan hacia la movilización y armonización de distintas clases de recursos, por ejemplo, recursos financieros desde la economía, recursos de poder bajo la forma de decisiones y apoyos políticos, recursos simbólicos bajo la forma de pautas culturales. Además de la diferenciación funcional, dicha armonización debe superar como obstáculo la "dominancia ecológica" (Jessop, 2000) de unos sistemas sobre otros, esto es, el hecho de que algunos de ellos tienden a influir con más frecuencia en las transformaciones de los demás en el contexto de una sociedad moderna capitalista. 


\subsection{Fallas de coordinación y problemas de desempeño}

En cada uno de estos niveles y en la coordinación de acciones que involucre los tres (sistemas, instituciones, individuos), se dan problemas de desempeño, esto es, situaciones en que no logran realizarse las expectativas de coordinación. Estos problemas son más probables en la medida que los distintos niveles se van complejizando a través de una profundización de los procesos de modernización como fue mostrado antes.

De no existir dispositivos que orienten los procesos de cambio, innovación y especialización que impone la modernización se corre el riesgo de liberar su potencial desintegrador. En este caso se dirá que se produce una falla de coordinación. Una falla de coordinación ocurre en espacios o situaciones donde a pesar de los procedimientos y mecanismos dispuestos para ello, la coordinación social no se realiza. Las fallas de coordinación se expresan de manera típica como una distancia entre la expectativa inicial de coordinación y el resultado final de la misma. En espacios de coordinación estructurados en torno a una determinada función, como los sistemas de protección social, esto puede denominarse un problema de desempeño. Estos problemas se manifiestan al menos de tres modos: como problemas para convocar a los agentes al espacio de coordinación en el nivel micro, como problemas de realización de las pautas de acción promovidas en el nivel funcional, o como problemas de orientación para reunir los recursos requeridos en el nivel sistémico. En cada caso, la consecuencia es un aumento de la incertidumbre, y de la impredecibilidad de las acciones, afectando el cometido de los sistemas y agentes involucrados en sus respectivas funciones ${ }^{3}$.

En situaciones de este tipo no es raro observar soluciones en que se recrean escenarios de menor complejidad en vez de hacerse cargo de la ya existente. Es el caso del recurso a pautas de acción autoritarias, por ejemplo. En el límite, puede esperarse que en un contexto de constantes fallas de coordinación los agentes y sistemas involucrados opten sistemáticamente por emplear soluciones de tipo egoísta. Si actuar en conjunto con otro o de acuerdo a lo prescrito trae asociado un alto riesgo por la imposibilidad de anticipar respuestas y resultados, entonces se hace mejor actuar solo. Con esto se pierde el potencial asociado a la acción colectiva y las formas cooperativas de coordinación.

De este modo, en términos de los desafíos de coordinación, la disminución de la probabilidad de ocurrencia de fallas de coordinación pasa porque los mecanismos de coordinación: i) definan pautas de acción legítimas, ii) relacionen los recursos sistémicos pertinentes, y iii) aseguren el mantenimiento de las expectativas individuales y la articulación de las agencias involucradas.

3 Hay que distinguir las situaciones de fallas del carácter inherentemente problemático de la coordinación social. Es necesario recalcar que esta es de por sí altamente improbable dada la densidad de factores que intervienen en cada espacio. Su existencia debe, según esto, ser analizada como una posibilidad y no tomarse por descontada. Es más, la coordinación social se vuelve más improbable cuantas más sean las variables que intervienen en los espacios en los que se la busca producir. Por lo mismo, contextos de mayor complejidad implican problemas o desafíos de coordinación más importantes. 


\section{LA PROTECCIÓN SOCIAL COMO PROBLEMA DE COORDINACIÓN}

En el centro de la problemática de la política social está la coordinación de relaciones sociales complejas. Dicha complejidad se manifiesta en la necesidad de armonización de las distintas prestaciones o beneficios ofrecidos, la articulación de las estructuras que proveen dichas prestaciones $y$, al mismo tiempo, de los agentes que en ellas actúan, incluidos los propios beneficiarios. A continuación se definirá qué es lo que se entiende por protección social como parte de un problema de coordinación social y se analizarán sus requerimientos en función de una matriz de análisis elaborada a partir de los elementos teóricos revisados.

\subsection{Los sistemas de protección social y su forma de operar la coordinación}

En las sociedades modernas se ha denominado típicamente sistemas de protección social a una estructura encargada de manera específica de regular la inclusión social. Ésta se institucionaliza de forma relativamente duradera en una sociedad conformando un ámbito funcional distinguible -aunque no necesariamente diferenciado. Establece un espacio de coordinación que involucra sistemas, estructuras e instituciones proveedoras típicas y roles determinados para cada una de ellas. La organización de los sistemas de protección social se basa en la definición de beneficiarios y prestaciones a partir de "teorías de la sociedad justa" y en la medida que estos elementos definen los contextos institucionales de la protección social, en ellos se encuentran inscritos los intereses de los diversos actores involucrados en dicha definición. De este modo, la discusión política es particularmente importante, pues permite a los sistemas de protección social inscribirse en principios normativos (la igualdad, la equidad, la justicia) que justifican su desempeño.

Los mecanismos de protección social operan cuando las reglas que regulan la inclusión en un determinado ámbito funcional producen una exclusión. En las sociedades capitalistas modernas, el dinero constituye la principal -aunque no la única- forma de inclusión. Éste permite no sólo la reproducción social a través de una procuración económica, sino también otras formas de inclusión como son, por ejemplo, aquellas de tipo simbólico a por medio del consumo. La exclusión del mercado del trabajo supone de esta manera una situación de exclusión de diversas esferas sociales.

Los mecanismos de protección social generan una estructura de expectativas de inclusión en torno a una categoría social. Las personas son identificadas como sujetas a expectativas de protección social, y en tanto que tales incluibles en la dinámica de las comunicaciones de una determinada política o programa social.

De la diferencia temporal en las expectativas de inclusión generadas por los mecanismos de protección social puede distinguirse entre dispositivos de aseguramiento, esto es, que anticipan estados de exclusión futura como los incluidos típicamente en un sistema de pensiones, y dispositivos de compensación que actúan sobre exclusiones ya realizadas como aquellos que se observan en las políticas asistenciales (Madariaga y Pérez, 2009). Del alcance de los mismos puede distinguirse entre mecanismos de protección social formales, es decir, aquellos que son sancionados por el Estado y asegurados en sus expectativas de inclusión 
a través del derecho, y mecanismos de protección social informales, esto es, aquellos que operan en espacios locales y para un número reducido de personas y con una expectativa reforzada de manera informal o puramente moral.

Por último, los mecanismos de protección social coordinan probabilidades de inclusión a partir de las prestaciones ofrecidas por una serie de otros subsistemas, espacios o estructuras, entre los que destacan típicamente la familia, el Estado y el mercado ${ }^{4}$ (Esping-Andersen, 2000). Cada una de ellas se asienta sobre formas de coordinación distintas, lo que les otorga una particularidad a las expectativas de protección social que ofrecen. En último término, las posibilidades de cada una de estas estructuras de proveer prestaciones de protección social están contenidas en sus propios límites en tanto mecanismos de coordinación social.

\subsection{El diseño de sistemas de protección social y sus requerimientos de coordinación en los distintos niveles}

La distinción de tres niveles analíticos propuesta para observar los problemas de coordinación en la sociedad puede replicarse al interior mismo del ámbito funcional que definen los mecanismos de protección social. Esto significa que dentro de la política social también pueden analizarse desafíos de coordinación que ponen en relación variables sistémicas, institucionales y microsociales. Luego, el desempeño de la política social puede centrarse ya sea en la manera como resuelve los desafíos de coordinación de su organización interna -el nivel funcional propiamente tal-, o bien ampliarse a evaluar el vínculo entre este y la sociedad en la que se inserta. La matriz que se presenta a continuación pone en relación estos dos niveles de análisis (mecanismos de protección social y sociedad). Cada casilla representa la forma que asume la necesidad de coordinación entre uno de los niveles del mecanismo de protección social con el correspondiente nivel en el conjunto de la sociedad.

El desafío de un sistema de protección social consiste en i) alinear los intereses de las agencias particulares (fila III) con los objetivos societales en materia de protección social (fila I), ii) en coordinar de manera adecuada las prestaciones de protección social ofrecidas, y iii) en vincular sus resultados con el funcionamiento de otros subsistemas sociales y viceversa. Para esto, los sistemas de protección social ponen en juego una serie de dispositivos de coordinación. El objetivo de éstos es no sólo la armonización de las acciones en cada uno de los niveles -microsocial, funcional y sistémico- sino además la puesta en relación de los recursos, objetos, actores y formas de interacción de los tres.

\section{i) El nivel microsocial}

Desde el punto de vista de las agencias involucradas, el desempeño de un sistema de protección social pasa primero por la generación de estructuras de expectativas de inclusión socialmente legítimas que a la vez constituyan alternativas válidas desde el punto de vista

4 En la literatura contemporánea se ha venido destacando crecientemente la labor que pueden ejercer otras como la sociedad civil o "tercer sector" y la propia comunidad (Cf. Herrera y Castón, 2003). 


\section{CUADRO 1}

Matriz de análisis de mecanismos de protección social como problemas de coordinación

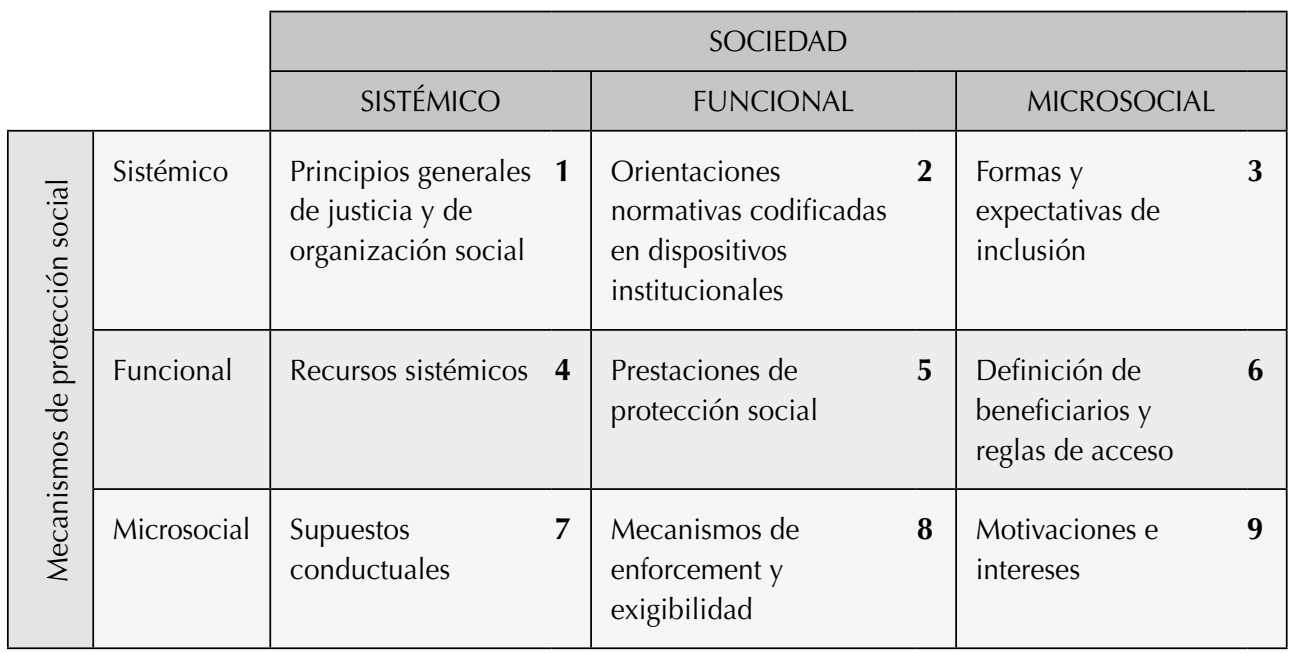

Fuente: elaboración propia.

de los agentes (casilla 3). Para esto deben generarse dispositivos destinados a aumentar las probabilidades de participación de las agencias en los sistemas de protección social formales, y disminuir las probabilidades de defección, esto es, de retirarse del juego.

Entre los dispositivos posibles de ser implementados con este objetivo pueden mencionarse: i) la adecuación de los mecanismos de protección social a supuestos conductuales. Éstos limitan las acciones posibles en materia de inclusión social y las hacen predecibles, inscribiéndolas en modelos de agentes típicos que ofrecen alternativas con sentido en el nivel individual (casilla 7); ii) el establecimiento de dispositivos que generan una motivación, o sea, siguiendo el concepto de illusio propuesto por Bourdieu, la sensación de "estar involucrado, de estar atrapado en el juego y por el juego. Estar interesado quiere decir aceptar que lo que acontece en un juego social determinado tiene un sentido, que sus apuestas son importantes y dignas de ser emprendidas" (Bourdieu y Wacquant, 1998: 78). Algunos dispositivos motivacionales típicos son la ampliación de las alternativas o posibilidades de elección o el establecimiento de recompensas y sanciones (casilla 9) y; iii) dado que los agentes no responden necesariamente a una racionalidad costo/beneficio, no siempre la existencia de recompensas o castigos es suficiente para generar las conductas esperadas. Se hace necesario el establecimiento de dispositivos de enforcement y exigibilidad que suponen la posibilidad de promover y obligar determinados cursos de acción, especialmente mediante la inscripción de los mismos en normas jurídicas o morales (casilla 8).

Este conjunto de elementos de diseño destinados al nivel microsocial puede tener dos tipos de énfasis (Pettit, 1993): uno que centra los dispositivos en quienes no actúan según lo prescrito, lo que supone poner el acento en las sanciones (deviant-centered strategy), y 
otro consistente en centrar la atención en las personas que se comportan según la norma, favoreciendo dispositivos como las recompensas y, especialmente, la selección adecuada de los beneficiarios mediante las reglas de acceso más apropiadas (compiler-centered strategy). La focalización de ciertas prestaciones o las estrategias de discriminación positiva en el acceso a servicios son algunos ejemplos. En ciertos casos las acciones se orientan a romper la barrera que inhabilita a los agentes para participar de estos espacios. El acompañamiento personalizado, capacitaciones o refuerzos a nivel psicosocial como en el caso de Chile Solidario son alternativas posibles (Cf. Raczynski, 2008).

\section{ii) El nivel funcional}

En el nivel funcional las acciones se orientan a la organización de las instituciones de protección involucradas. Esto, de manera tal de gobernar el conjunto de prácticas sociales y de operaciones sistémicas que intervienen hacia la consecución de un objetivo societal específico, en este caso el de la protección social. Además, se busca hacer efectiva la inclusión de los agentes -beneficiarios- en este ámbito funcional.

Para ello se requiere: i) obtener recursos desde otros sistemas bajo la forma de dinero, decisiones, patrones culturales. Aquí se pueden observar los requerimientos sistémicos para dar cumplimiento a las formas de inclusión ofrecidas (casilla 4); ii) coordinar las acciones que se desarrollan al interior del propio ámbito funcional que representa la protección social. Las acciones se orientan hacia la alineación de la racionalidad inscrita en las estructuras de protección social, lo que implica, a su vez, alinear las lógicas de los agentes que participan en la producción de dichas prestaciones. Para esto deben implementarse dispositivos de coordinación entre las instituciones y entre las prestaciones que conforman el sistema de protección de manera tal de evitar duplicaciones, de hacer más eficiente el uso de los recursos, entre otros (casilla 5); iii) finalmente, existe una definición respecto de quiénes son los sujetos de protección, esto es, aquellos sujetos susceptibles de desarrollar las expectativas de inclusión que el sistema de protección define. Esto se logra mediante el establecimiento de reglas de acceso que expresan una o un conjunto de acciones a ser emprendidas por los sujetos para acceder a las prestaciones ofrecidas o bien, una condición que los hace meritorios de ser incluidos como beneficiarios del programa (casilla 6).

\section{iii) El nivel sistémico}

En el nivel sistémico las acciones deben orientarse hacia las posibilidades de inclusión de los agentes en distintos subsistemas relevantes, por ejemplo, el de la política o la economía.

Para ello necesitan determinadas orientaciones normativas bajo la forma de principios que justifiquen las ofertas de inclusión y los resultados obtenidos, y que guíen su codificación en determinados dispositivos institucionales. Es así como la norma de asignación y distribución de recursos que el sistema define puede tener sentido para los demás subsistemas. Con esto se logrará un cierto grado de integración de los diversos sistemas más allá de su principio de autonomía y clausura operacional (casilla 2). 
En la definición de una determinada forma de inclusión asociada a cada acción de protección se determinan también los subsistemas involucrados en su realización. Esta forma de inclusión puede ser unidimensional o multidimensional. Ejemplos del primer caso son las transferencias monetarias que sólo intervienen directamente en una inclusión económica, sin desmedro del hecho que esto pueda, a modo de consecuencias no esperadas, favorecer formas de inclusión en otros ámbitos como, por ejemplo, acceder a ciertas prestaciones en el ámbito de la salud. Si estas transferencias se acompañan además de la consagración de ciertos derechos en salud, entonces la inclusión se produce además en el sistema legal. De esta manera se diversifican las expectativas posibles de ser realizadas.

Cabe señalar que esta matriz se propone para el análisis de cualquier política social. Por lo mismo, según cual sea el caso y los objetivos, distinto será el uso que de ella pueda hacerse. Por ejemplo, si bien cada casilla de la matriz representa la forma que asume la necesidad de coordinación entre uno de los niveles del mecanismo de protección social con el correspondiente nivel en el conjunto de la sociedad, según desde donde se dirija la mirada, distintas serán las preguntas a formular. Aquí se propone utilizarla en dos sentidos. Primero, como una autoobservación de la política social sobre sus propios procesos (fila II). Segundo, para observar la manera como coordina sus orientaciones y prestaciones con las definiciones socialmente construidas respecto del tema de la inclusión, y los requerimientos sistémicos que definen las posibilidades de realización de las expectativas que genera entre sus beneficiarios (filas I y III).

Es importante recalcar que cualquier acción de protección social se inscribe simultáneamente en los tres niveles. Se deduce a partir de esto que el cumplimiento de las expectativas contenidas en un sistema de protección social requiere no sólo de la armonización de las acciones en cada uno de los niveles, sino además de la acción de dispositivos que pongan en relación los recursos, objetos, actores y formas de interacción predominantes en los tres. Ello puede entenderse bajo el concepto de sinergias (Repetto, 2009), que capta los desafíos de coordinación en cada nivel y entre los distintos niveles. En el nivel microsocial se requiere de sinergias de intereses y motivaciones que permitan sustentar formas cooperativas de coordinación (9); entre el nivel microsocial y el funcional se trata de sinergias cognitivas que promueven el intercambio de información y conocimiento entre las partes (6 y 8); en el nivel funcional de sinergias de procesos y actividades necesarias para el establecimiento de acciones estratégicas concertadas y prestaciones recíprocas entre los agentes y estructuras involucrados (5); entre el nivel funcional y el sistémico sinergias de recursos que permitan las articulaciones y complementariedades entre los diversos recursos sistémicos requeridos a través de determinados dispositivos (2 y 4). A nivel sistémico, por último, se necesita de sinergias de gobierno que aseguren un cierto grado de integración de los diversos sistemas más allá de su principio de autonomía y clausura operacional (1).

\section{COMENTARIOS FINALES}

Abordar desde la política social la complejidad que supone hoy el problema de la inclusión requiere complejizar al mismo tiempo la mirada que se tiene sobre la misma política social 
y los ámbitos en los que ésta interviene. La apuesta ha sido en este texto la extensión del concepto de coordinación social hacia el análisis de las políticas de protección social. El resultado de ello ha sido una herramienta que permite observar los desafíos de coordinación que éstas enfrentan en distintos ámbitos y niveles.

La matriz propuesta puede utilizarse para el análisis de cualquier componente del sistema de protección social o, más generalmente, del sistema en su conjunto si se lo entiende como un ámbito funcional delimitado. De la misma manera, según desde dónde se mire o de cuáles sean los objetivos del análisis, distintas serán las casillas o ámbitos sobre los que centrar la atención. Un punto crítico a enfatizar es el hecho que todas las casillas deben ser consideradas. Por lo general, tiende a ocurrir que los análisis de diseño o desempeño de políticas o programas social centran su atención en las casillas centrales de la matriz (aquellas que resultan del cruce entre el nivel funcional del sistema de protección y la sociedad), desatendiendo -o tomando por supuestos invariables- aquellas que remiten a los desafíos de coordinación de la política con los niveles microsociales o sistémicos. Esta matriz pretende iluminar con una propuesta de análisis aquellas áreas cuya importancia es frecuentemente subvalorada. Es necesario destacar que sea cual sea el uso que se le dé, la descomposición analítica de los desafíos de coordinación que enfrenta una intervención en el ámbito de la inclusión social puede permitir identificar más claramente los nodos críticos de su desempeño y, eventualmente, las posibles soluciones a implementar.

Por ejemplo, en el caso de los sistemas de pensiones de capitalización individual, como es el chileno, existen numerosos trabajos empíricos que muestran los distintos problemas y fallas de coordinación en diferentes niveles. En el sistémico se han estudiado las relaciones entre el sistema de capitalización individual y el mercado de capitales, y la manera cómo los recursos financieros provenientes de las cotizaciones pueden ser canalizados hacia formas de inversión productiva que permitan aumentar el crecimiento económico, pudiendo encontrarse evidencias divergentes (Corbo y Schmidt-Hebbel, 2003; Uthoff, 2001). En el nivel funcional, el mayor énfasis se ha puesto en la conformación adecuada de un mercado de administradoras, cuya tendencia ha sido a la concentración y la falta de competencia (Arrau y Valdés, 2002; Valdés, 2005). Sin embargo, pese que la estructura del sistema de pensiones chileno hace que la forma de inclusión que promueve descanse principalmente en las decisiones de agentes individuales y que, en consecuencia, los problemas de coordinación del nivel microsocial sean particularmente importantes, los programas de investigación en este nivel son menos frecuentes ${ }^{5}$.

Los desafíos de coordinación de los sistemas de pensiones en el nivel microsocial consisten en coordinar las respuestas de los individuos a las ofertas de protección en la vejez, de manera de ajustar los objetivos individuales y sociales de procuración económica durante esta etapa de la vida. La inscripción de un sistema de pensiones en un principio normativo permite, en este contexto, incorporar hipótesis respecto de cómo actuarán los agentes ante las ofertas institucionalizadas de protección social. En la medida que estos

5 Casos especiales son los de Berstein y Rodríguez (2005) y particularmente Madariaga y Pérez (2009). 
supuestos y los dispositivos diseñados en función de ellos se ajusten a los comportamientos reales de las personas, debiera aumentar la probabilidad de coordinación de las respuestas individuales.

Buena parte de las reformas realizadas el año 2008 al sistema de pensiones chileno estuvo efectivamente dirigida a mejorar la coordinación del sistema en este nivel. Más específicamente, se centró en el establecimiento de incentivos y el aumento de la información disponible para la toma de decisiones. Sin embargo, en los análisis que fundamentaron estas reformas el comportamiento de los agentes no fue considerado dentro de una problemática general de coordinación, sino más bien ingresado al modelo como una constante. Ésta fue construida sobre la base de diversos supuestos conductuales los que, durante el último tiempo, han sido objeto de duros cuestionamientos por no observarse en la práctica ${ }^{6}$. Este punto es particularmente crítico en un contexto en el que el desempeño del sistema -en este caso el monto de las pensiones que dispone cada persona a la edad de la jubilacióndepende de la adecuación de las prácticas reales a los supuestos conductuales sobre los que está construido. Éstos operan, efectivamente, como el mecanismo que vincula intereses individuales y selecciones sociales en este ámbito. Cuando estos son errados, se produce una falla de coordinación que se expresa en un incumplimiento de las expectativas de inclusión ofrecidas inicialmente por el sistema de pensiones.

Este breve análisis pretende ilustrar el modo en que la aplicación de la matriz propuesta podría contribuir a identificar problemas específicos de coordinación en los sistemas de protección social y, eventualmente, a proponer reformas. En el caso del sistema de pensiones, siguiendo el ejemplo, modificaciones orientadas a asegurar el vínculo entre las acciones en el nivel microsocial, las dinámicas institucionales y las orientaciones normativas en las que se insertan.

\section{BIBLIOGRAFÍA}

Abramovich, Víctor (2006): "Una aproximación al enfoque de derechos en las estrategias y políticas de desarrollo", Revista de la CEPAL, № 88, Santiago, pp. 35-50.

Amable, Bruno (2003): The Diversity of Modern Capitalism, Oxford University Press, Oxford.

Arrau, Patricio y Salvador Valdés (2002): "Para desconcentrar los fondos de pensiones y aumentar la competencia en su administración", Estudios Públicos, № 85, Santiago.

Berstein, Solange y José Luis Rodríguez (2005): "Sensibilidad de la demanda con consumidores desinformados: el caso de las AFP en Chile", Documentos de Trabajo, № 4, Superintendencia de AFP (SAFP), Santiago.

Boltanski, Luc y Laurent Thévenot (1991): De la justification. Economies de la grandeur, Gallimard, Paris.

6 Al respecto, junto con la literatura sugerida en la nota anterior para el caso de Chile, vale la pena revisar literatura referente a otros países. Para Estados Unidos, Chan y Huff Stevens (2003) y Loewenstein (1999). De particular interés es la literatura que estudia el sistema sueco que incorpora varias premisas sobre las que actúa el sistema chileno (Cf. Cronqvist y Thaler, 2004). 
Castel, Robert (2006) : La metamorfosis de la cuestión social, Paidós, Buenos Aires.

Chan, Sewin, y Ann Huff S. (2003): "What you don't know can't help you?", NBER Working Paper, 10185.

Chernilo, Daniel (2006): “La teoría de la coordinación social en sociedades diferenciadas. La teoría de los medios simbólicamente generalizados es Parsons, Luhmann y Habermas", en Ignacio Farías y José Ossandón (eds.): Observando sistemas: nuevas apropiaciones y usos de la teoría de Niklas Luhmann, RIL, Santiago.

Christensen, Tom y Per Lægreid (2005): "El Estado fragmentado: los retos de combinar eficiencia, normas institucionales y democracia", Gestión y política pública, № 14, Vol. 3, pp. 557-598.

Corbo, Vittorio y Klaus Schmidt-Hebbel (2003): Efectos macroeconómicos de la reforma de pensiones en Chile, mimeo.

Cronqvist, Henrik, y Richard H. Thaler (2004): “Design Choices in Privatized Social-Security Systems: Learning from the Swedish Experience", American Economic Review, № 94, Vol. 2, pp. 424-428.

Esping-Andersen, Gösta (1989): "The three political economies of the welfare state", The Canadian Review of Sociology and Anthropology, № 26, pp. 10-36.

(2000): Fundamentos sociales de las economías postindustriales, Ariel, Barcelona.

Fajnzylber, Eduardo y Andrea Repetto (2007): "Instrumentos alternativos para la protección social", Serie Estudios Socio/Económicos, No 33, CIEPAN, Santiago.

Ffrench-Davis, Ricardo (2003): Entre el neoliberalismo y el crecimiento con equidad. Tres décadas de política económica en Chile (3a ed.), JC Sáez editor, Santiago.

Filgueira, Carlos y Fernando Filgueira (2002): "Models of Welfare and Models of Capitalism: The Limits of Transferability", en Evelyne Huber (ed.): Models of Capitalsm. Lessons for Latin America, The Pennsylvania Stata University Press, Pennsylvania.

Garnier, Leonardo (2000): Función de coordinación de planes y políticas, Instituto Latinoamericano y del Caribe de Planificación Económica y Social, ILPES, Santiago.

Herrera, Manuel y Pedro Castón (2003): Las políticas sociales en sociedades complejas, Ariel, Barcelona.

Hollingsworth, Rogers, y Boyer Robert (1997): "Coordination of economic actors and social systems of production", en Rogers Hollingsworth, y Robert Boyer (eds.): Contemporary capitalism. The embeddedness of institutions, Cambridge University Press, Nueva York, pp. 1-47.

Jessop, Bob (2002): Governance and Metagovernance: On Reflexivity, Requisite Variety and Requisite Irony, Department of Sociology, University of Lancaster, Lancaster, UK, mimeo.

(2000): Institutional (Re)Turns and the Strategic-Relational Approach, Department of Sociology, University of Lancaster, Lancaster, UK, mimeo.

(1999): The Governance of Complexity and the Complexity of Governance: Preliminary Remarks on some Problems and Limits of Economic Guidance, Department of Sociology, University of Lancaster, Lancaster, UK, mimeo.

Larrañaga, Osvaldo y Rodrigo Herrera (2008): "Los recientes cambios en la desigualdad y la pobreza en Chile", Estudios Públicos, № 109, Santiago, pp. 149-186.

Lechner, Norbert (1997): “Tres formas de coordinación social", Revista de la CEPAL, № 61, Santiago, pp. 7-17. 
Loewenstein, George (1999): "Is more choice always better?", Social Security Brief, № 7.

Luhmann, Niklas (2002): La teoría política en el estado de bienestar, Alianza editorial, Madrid. (1998): Complejidad y modernidad. De la unidad a la diferencia, Trotta, Madrid.

Madariaga, Aldo y Nicolás Pérez (2009): “Nuevas aproximaciones para el análisis de mecanismos de protección social. La noción de desempeño institucional y su aplicación al sistema de pensiones chileno", Revista MAD, № 20, Santiago, pp. 1-42.

Marcel, Mario y Elizabeth Rivera (2008): "Regímenes de bienestar en América Latina", en Eugenio Tironi (ed.): Redes, Estado y mercado. Soportes de la cohesión social latinoamericana, Uqbar, Santiago.

Messner, Dirk (1999): “Del Estado céntrico a la 'sociedad de redes'. Nuevas exigencias a la coordinación social", en Norbert Lechner, René Millán y Francisco Valdés: Reforma del Estado y coordinación social, Plaza y Valdés, México, pp. 77-121.

Millán, René (1999): "Problemas generales y particulares de la coordinación social”, en Norbert Lechner, René Millán y Francisco Valdés: Reforma del Estado y coordinación social, Plaza y Valdés, México, pp. 55-76.

Pettit, Pascal (1993): "Institutional design and rational choice", en Robert Goodin (ed.): The Theory of Institutional Design, Cambridge University Press, Cambridge.

Polanyi, Karl (2003): La gran transformación. Los orígenes políticos y económicos de nuestro tiempo (2da ed.), Fondo de Cultura Económica, México.

PNUD (2000): Desarrollo Humano en Chile. Más sociedad para gobernar el futuro, Programa de las Naciones Unidas para el Desarrollo, Santiago.

(2002): Desarrollo Humano en Chile. Nosotros los chilenos: un desafío cultural, Programa de las Naciones Unidas para el Desarrollo, Santiago.

(2009): Desarrollo Humano en Chile. La manera de hacer las cosas, Programa de las Naciones Unidas para el Desarrollo, Santiago.

Raczynski, Dagmar (2002): “Equidad, inversión social y pobreza. Innovar en cómo se concibe, diseña y gestionan las políticas y los programas sociales", Documento preparado para el seminario Perspectivas innovativas en política social. Desigualdades y reducción de brechas de equidad, MIDEPLAN-CEPAL, Santiago, mayo 23 y 24.

(2008): Sistema Chile Solidario y la Política de Protección Social de Chile. Lecciones del pasado y agenda para el futuro, CIEPLAN, Santiago.

Repetto, Fabián (2009): Coordinación de políticas sociales: abordaje conceptual y revisión de experiencias latinoamericanas, mimeo.

Riesco, Manuel (2009): "El modelo social chileno comienza a cambiar", Revista Internacional del Trabajo, № 128, Vol. 3.

Schkolnick, Mariana y Josianne Bonnefoy (1994): Una propuesta de tipología de las políticas sociales en Chile, UNICEF, Santiago.

Stallings, Barbara (2001): "Las reformas estructurales y el desempeño socioeconómico", en Ricardo Ffrench-Davis y Barbara Stallings (eds.): Reformas, crecimiento y políticas sociales en Chile desde 1973, CEPAL-LOM, Santiago. 
Théret, Bruno (2005): "De la comparabilité des systémes nationaux de protection sociale dans les societés salariales. Essai d'analyse structurale", Recherche y Régulation Working Papers.

Torche, Florencia (1996): "Exclusión social y pobreza: implicancias de un nuevo enfoque" en Paula Barros, Danae de los Ríos y Florencia Torche: Lecturas sobre la exclusión social, OIT, Santiago.

Uthoff, Andras (2001): "La reforma del sistema de pensiones en Chile. Desafíos pendientes", Serie financiamiento del desarrollo, № 112, Comisión Económica para América Latina y el Caribe (CEPAL), Santiago.

Valdés, Salvador (2005): "Para aumentar la competencia entre las AFP", Estudios Públicos, № 98, Santiago.

Willke, Helmut (2007): Smart Governance. Governing the Global Knowledge Society, The University of Chicago Press, Chicago.

(1993): "Formas de autoorientación de la sociedad", en Teoría de sistemas de las sociedades desarrolladas. Dinámica y riesgo de la autoorganización social moderna (Trad. Aldo Mascareño), Juventa Verlag, München. 